\title{
On the Theory of the Plasma Capillary Effect
}

\author{
A. A. Bredikhin ${ }^{a}$ and E. B. Kulumbaev ${ }^{a, *}$ \\ ${ }^{a}$ Belgorod State National Research University, Belgorod, Russia \\ *e-mail: kulumbaev@bsu.edu.ru \\ Received December 19, 2019; revised December 19, 2019; accepted October 14, 2020
}

\begin{abstract}
From the condition of the minimum energy of a three-phase system in an equilibrium stable state, it is shown that the plasma capillary effect occurs due to the electrification of the meniscus when the liquid comes into contact with the plasma. The presence of an electric charge on the meniscus leads to an increase in the wettability of the capillary walls by the liquid. It was additionally established that a second stationary, but unstable state of the system appears in this case. The calculation results are consistent with the observational data.
\end{abstract}

DOI: $10.1134 / \mathrm{S} 0018151 \mathrm{X} 20060061$

\section{INTRODUCTION}

The work [1] reports on the discovery of a new physical effect consisting of an increase in the height of the liquid column in a capillary when the gas-discharge plasma touches the meniscus of the liquid and is called the plasma capillary effect. According to [1], the height of the liquid column (30\% aqueous solution of copper sulfate) increases abruptly at the moment of the spark discharge in the gap between the needle electrode and the meniscus of the liquid, which is initiated by a single, high-voltage, nanosecond pulse, and then the height of the column does not change, at least, for a few hours. According to the authors of [1], the liquid rises due to the electrification of the meniscus by a spark discharge, which leads to an increase in the wettability of the capillary walls by the liquid. However, this conclusion was based only on a qualitative discussion of the results of their own observations and similar experiments described in a number of other works (references are given in [1]). Therefore, the goal of this work is to substantiate the mechanism of the plasma capillary effect proposed in [1] based on a simple mathematical model.

\section{MODEL}

A three-phase system "liquid-solid (capillary)gas" limited by the walls of a cylindrical capillary is considered. It is believed that the liquid wets the capillary, and, thus, the capillary effect is realized: when the capillary is lowered into in a wide vessel, the liquid in the capillary rises relative to the main liquid level in the vessel (Fig. 1).

It is also taken into account that the curved surface of the capillary liquid can be charged with electricity. The meniscus is considered in the approximation of the charged surface of an "ideal" liquid conductor, inside which the electric field is disregarded. The applicability of such a simplified representation of an electrified liquid (water and aqueous solutions) is justified in electrostatics by its "large" dielectric constant and in the electrohydrodynamics of charged surfaces by the rapid relaxation of charges [2]. The electric field from the gas side is perpendicular to the meniscus at any point. Otherwise, as experience shows, fluid motion occurs [2]. The tangential component of the field on the meniscus is zero due to the distribution of electric charges ensuring its equipotentiality. Such an electric field leads to an electric pressure drop, which is equivalent to an increase in the surface tension of the liquid for a concave meniscus. As a result, the height of the liquid column in the capillary can increase.

The stationary steady state of the considered system "liquid-capillary-gas" corresponds to the minimum of its energy, which is composed of the surface

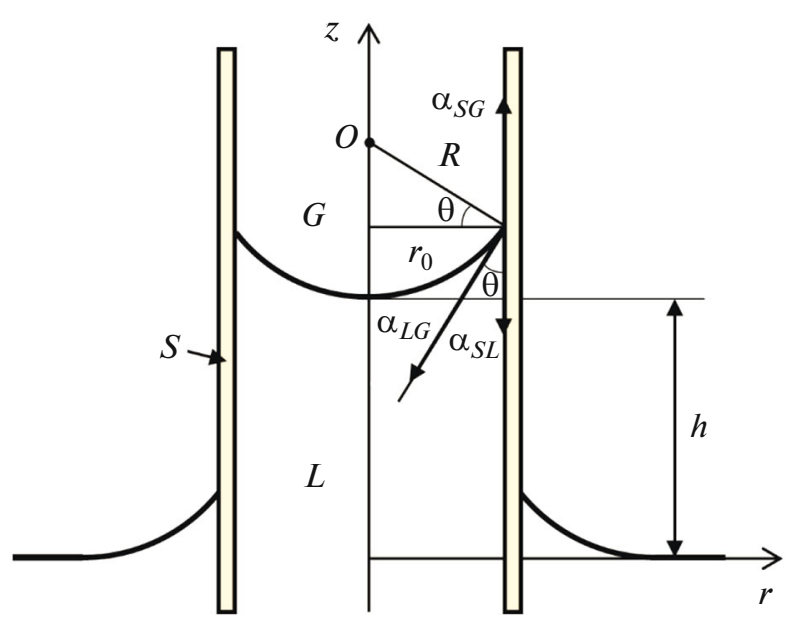

Fig. 1. Geometry of the capillary rise of the wetting liquid. 
energy, the potential energy of the liquid column in the capillary in a uniform gravitational field, and the interaction energy of electric charges located on the equipotential meniscus:

$$
E=\alpha_{S L} A_{S L}+\alpha_{S G} A_{S G}+\alpha_{L G} A_{L G}+m g h_{C}+q^{2} / 2 C,
$$

where $\alpha$ is the coefficient of surface tension at the contact boundary with the area $A$ of the two phases indicated by subscripts; $L$ is a liquid; $S$ is a capillary; $G$ is a gas (Fig. 1); $m$ is the mass of the liquid column in the capillary; $h_{C}$ is the height of the center of gravity of the liquid column relative to the main liquid level in a wide vessel; and $q$ and $C$ are the external electric charge and electric capacity of the meniscus.

In the approximation of the axisymmetric meniscus, the problem of energy minimization is simplified if we take into account in (1) the conditions of mechanical equilibrium of the meniscus apex and the common line of contact of the three phases on the capillary wall. These conditions for an uncharged meniscus, respectively, are expressed with the Laplace formula (the equality of capillary and hydrostatic pressures at the apex of the meniscus, the main radii of curvature of which are the same) and by Young's formula (the balance of the component forces tangential to the capillary wall acting on a unit element of the contact line from the side of three interface surfaces (Fig. 1)). For a charged meniscus, the Laplace formula is supplemented with allowance for the electrical pressure; therefore, the conditions of mechanical equilibrium are written in the form

$$
\frac{2 \alpha_{L G}}{R}+\frac{\sigma_{0}^{2}}{2 \varepsilon_{0}}=\rho g h, \quad \alpha_{S G}=\alpha_{S L}+\alpha_{L G} \cos \theta .
$$

Here, $\rho$ is the density of the liquid (the density of the gas is disregarded in comparison with $\rho$ ); $h$ and $R$ are the height and radius of curvature of the meniscus at its apex; $\sigma_{0}$ is the surface density of the electric charge at the top of the meniscus; and $\theta$ is the contact angle (Fig. 1).

It is seen from (2) that the presence of electricity at the meniscus is equivalent to an increase in the surface tension coefficient at the apex. To estimate the order of the increase $\alpha_{L G}$ with respect to the electrical charge, the meniscus is assumed to be hemispherical with a uniform distribution $q$ on its surface. Then the share $\gamma=R \sigma_{0}^{2} /\left(4 \varepsilon_{0} \alpha_{L G}\right)=q^{2} /\left(16 \pi^{2} \varepsilon_{0} r_{0}^{3} \alpha_{L G}\right)$ is the ratio of the characteristic values of the electric and surface energies of the charged hemisphere, and $r_{0}$ is the radius of the capillary. For example, for water in a capillary with a radius of $1 \mathrm{~mm}$, we get $\gamma \sim\left(10^{9} q\right)^{2}$, i.e., at $q=1 \mathrm{nC}, \alpha_{L G}$ doubles (effectively). Therefore, in order to avoid the self-consistent formulation of mechanical and electrostatic problems for a liquid column in a capillary, which is necessary for a rigorous approach, the electrification of the meniscus is further assumed to be small so that the contribution of the electric pressure can be disregarded in (2) and it is assumed that $R h=a^{2}$ and that $a=\sqrt{2 \alpha_{L G} / g \rho}$ is the capillary constant for the top of the meniscus. Young's formula is used to exclude $\alpha_{S G}-\alpha_{S L}$ from (1) by replacing $\alpha_{L G} \cos \theta_{Y}$, where $\theta_{Y}$ is the contact angle in the case of an uncharged meniscus, similar to the theory of electrowettability on a dielectric (e.g., [3]). In this case, the presence of electricity on the meniscus is taken into account only in the expression for the energy (1) for the considered system, the minimum of which is realized at some contact angle $\theta$ different from $\theta_{Y}$.

Further simplification of the model consists of an approximation of the meniscus with a spherical segment, the acceptability of which is discussed below. Then, taking into account the geometric formulas for a constant radius of curvature of $R=r_{0} / \cos \theta$ of the spherical segment, the areas of the interphase surfaces are $A_{L G}=2 \pi R h_{0}, A_{S L}=2 \pi r_{0} H$, and $A_{S G}=A_{0}-A_{S L}$ ( $h_{0}=R(1-\sin \theta)$ is the segment height, $H=h+h_{0}$, and $A_{0}$ are the area of the inner surface of the capillary), the height $h_{C}$ is the center of gravity on the axis of an axisymmetric homogeneous ( $\rho=$ const) of the liquid column as the center of gravity of its volume $V$ from the formula $V h_{C}=\pi r_{0}^{2} H^{2} / 2-V_{0}\left(h+R-\pi r_{0}^{4} / 4 V_{0}\right)$, where $V_{0}=\pi h_{0}^{2}\left(R-h_{0} / 3\right)$ is the volume of the spherical segment, and formulas for the electrical capacity $C=4 \varepsilon_{0} R(\pi / 2-\theta+\cos \theta)$ of the spherical segment [4]. The energy expression (1) is reduced to the dependence of $E$ on the contact angle $\theta$ in the form of

$$
\begin{gathered}
E^{\prime}=-2 \frac{\cos ^{2} \theta+b(1-\sin \theta)}{b \cos \theta} \cos \theta_{Y}+2 \frac{1-\sin \theta}{\cos ^{2} \theta} \\
+\frac{1}{b}\left(\frac{\cos ^{2} \theta+b(1-\sin \theta)}{\cos \theta}\right)^{2} \\
-\frac{2}{3} \frac{(1-\sin \theta)^{2}(2+\sin \theta)\left(\cos ^{2} \theta+b\right)}{\cos ^{4} \theta}+\frac{b}{2} \\
+\gamma \frac{2 \pi \cos \theta}{\pi / 2-\theta+\cos \theta} .
\end{gathered}
$$

Here, $E^{\prime}=\left(E-\alpha_{S G} A_{0}\right) /\left(\alpha_{L G} \pi r_{0}^{2}\right)$ is the variable part of the dimensionless energy of the considered system; $b=\left(r_{0} / a\right)^{2}=\rho g r_{0}^{2} /\left(2 \alpha_{L G}\right)$ is the square of the ratio of the capillary radius to the capillary constant or the ratio of potential energy $\rho g \pi r_{0}^{4} / 2$ of a straight, circular, cylindrical column of liquid with the same radius and height equal to $r_{0}$ in the field of gravitation to the surface energy $\alpha_{L G} \pi r_{0}^{2}$ of its upper base (note that $b$ is half of the Bond number). 
Thus, the problem of minimizing energy (1) is reduced to the determination of the contact angle $\theta$ corresponding to the minimum of function (3).

\section{ANALYSIS}

When $b \ll 1$, expression (3) for energy is noticeably simplified:

$$
E^{\prime}=\frac{\left(\cos \theta-2 \cos \theta_{Y}\right) \cos \theta}{b}+\gamma \frac{2 \pi \cos \theta}{\pi / 2-\theta+\cos \theta},
$$

and its minimum is realized at the contact angle $\theta$, which satisfies the equation

$$
\begin{gathered}
\frac{\sin \theta\left(\cos \theta-\cos \theta_{Y}\right)}{b} \\
+2 \pi \gamma \frac{(\pi-2 \theta) \sin \theta-2 \cos \theta}{(\pi-2 \theta+2 \cos \theta)^{2}}=0 .
\end{gathered}
$$

It is seen that $\theta_{Y}$ is the root of this equation only for an uncharged meniscus. This means that the approximation of the meniscus by a spherical segment is acceptable if two conditions are met simultaneously: $b \ll 1$ and $\gamma=0$. In particular, the famous Jurin's law, $r_{0} h=a^{2} \cos \theta_{Y}$, which was obtained for a spherical meniscus from the conditions of mechanical equilibrium of the apex of an uncharged meniscus and the common line of contact of three phases on the capillary wall, strictly speaking, does not agree with the condition of the minimum energy (1) of the liquid column in a capillary in a stationary state for a spherical meniscus. Then, the closeness of the value $\theta$ corresponding to the minimum (3) to $\theta_{Y}$ is taken as an a posteriori estimate of the acceptability of the approximation of an uncharged meniscus by a spherical segment.

Minimization (4), in contrast to the analogous problem for (3), has two parameters; therefore, transcendental equation (5) is solved numerically in the interval $\theta \in(0, \pi / 2)$ at a fixed contact angle $\theta_{Y}$ for a set of parameter values $\gamma b$. Figure 2 shows the calculation results.

It can be seen from Fig. 2 that there are sets of parameter values at which two states of a three-phase system, which are extreme in energy, are realized: stable ones with a minimum energy (e.g., state $A$ is at $\theta=52^{\circ}$ at $\gamma b=0.5$ and $\theta_{Y}=60^{\circ}$ ) and unstable ones with a maximum energy (state $B$ is at $\theta=23^{\circ}$ for the same $\gamma b$ and $\theta_{Y}$ ).

\section{RESULTS AND DISCUSSION}

A numerical estimate of the effect of meniscus electrification on the height of the liquid in the capillary was carried out based on the experimental data [1], according to which the height of a $30 \%$ aqueous solution of copper sulfate prior to meniscus electrification was about $6 \mathrm{~mm}$ in a capillary with a radius of $0.85 \mathrm{~mm}$. After the discharge pulse, it increased abruptly by $0.5-3 \mathrm{~mm}$. Coefficient $\alpha_{L G}$ was taken as

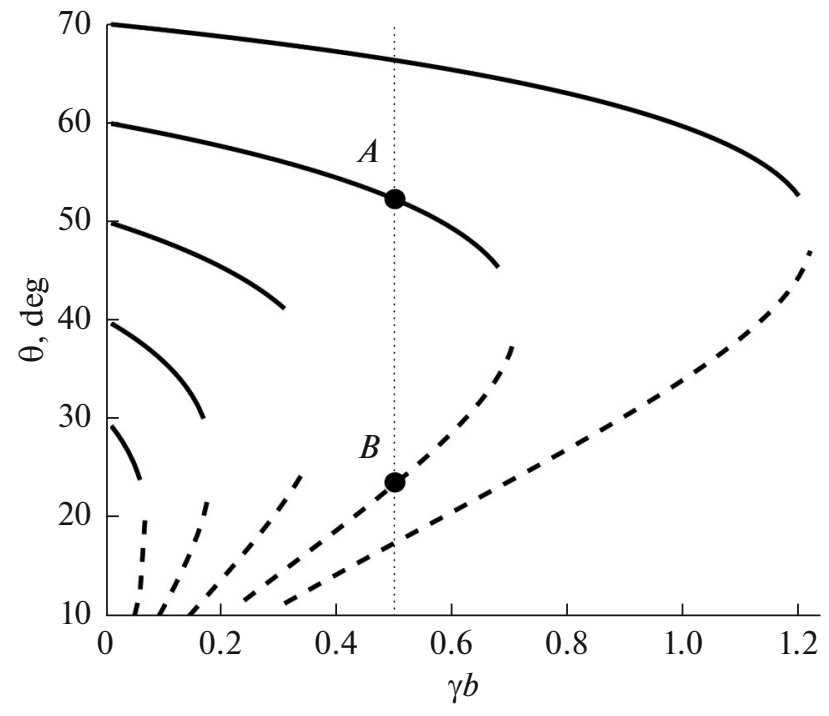

Fig. 2. Position of the points of energy extrema (4) of the three-phase system "liquid-capillary-gas" with respect to the parameters $\gamma b$ and $\theta_{Y}$ : solid lines indicate stable, stationary states with minimum energy, and dashed lines indicate unstable states with maximum energy.

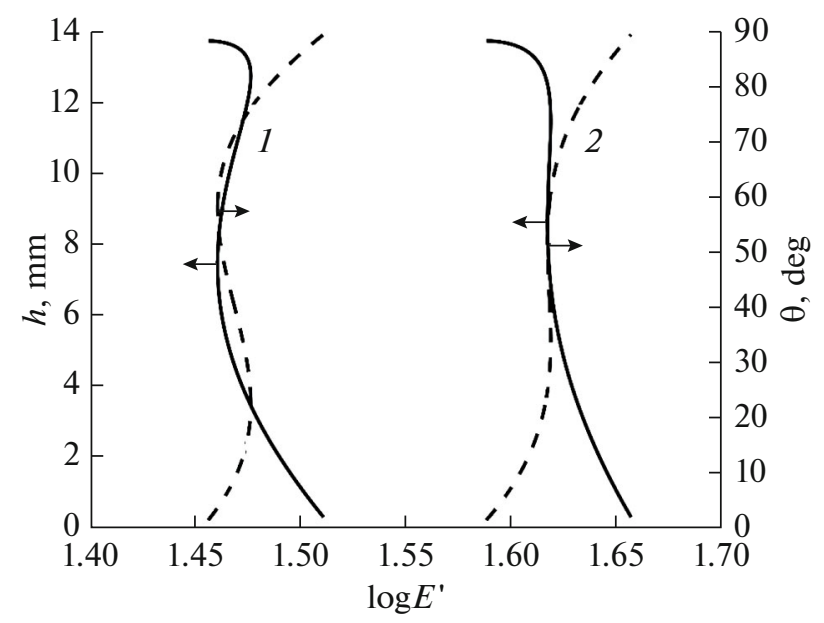

Fig. 3. Column height (solid lines) and contact angle (dashed lines) with respect to the variable part of the energy $E$ " of the three-phase system "copper sulfate aqueous solution-glass capillary-air," when charged on a spherical meniscus (capillary radius of $0.85 \mathrm{~mm}) q=0.8$ (1) and $1 \mathrm{nC}(2)$.

equal to $74 \mathrm{mN} / \mathrm{m}$ according to [5]. Then, $b \approx 0.06$, and the contact angle corresponding to the height of the column before electrification, according to Juren's formula, was $\theta_{Y}=64^{\circ}$. Figure 3 shows the results of calculation of these data with formula (3) as the dependences $\theta\left(E^{\prime}\right)$ and $h\left(E^{\prime}\right)$ for two values of the meniscus charge.

The calculation shows that the minimum $E^{\prime}$ of a three-phase system prior to meniscus electrification corresponds to $\theta$ and $h$, which practically coincide with the experimental values of these quantities. Therefore, the approximation of the meniscus by a spherical segment is acceptable in the considered case. 
At a $0.5 \mathrm{nC}$ charge on the meniscus, the wettability increases: the contact angle decreases by $2^{\circ}$, and the column height increases by $0.5 \mathrm{~mm}$. Moreover, depending $E^{\prime}(\theta)$ (and $E^{\prime}(h)$, respectively), an additional extremum is realized: it is a local maximum, which indicates the emergence under these conditions of a second stationary but unstable state of the system. The energy of an unstable state exceeds the energy of a stable state by $35 \%$. It can be seen in Fig. 3 that with an increase in the meniscus charge to $0.8 \mathrm{nC}$, the contact angle of the stable state of the system becomes equal to $57^{\circ}$, the height is $7.4 \mathrm{~mm}$, and the parameters of the stable and unstable system states (extrema $E^{\prime}$ ) approach each other. Further, the noted tendencies persist; thus, $h=8.6 \mathrm{~mm}$ and the energies of states differ only by $0.3 \%$ at a charge of $1 \mathrm{nC} \theta=51^{\circ}$. At $q>1 \mathrm{nC}$, there are no stationary states in the considered system.

The calculated value of the additional rise of capillary liquid by $2.8 \mathrm{~mm}$ due to the charge on the meniscus agrees with the observational data [1].

The existence within the framework of the considered model of a critical electric charge, above which the system has no stationary states, corresponds to the general pattern of behavior of charged surfaces of liquids: when a critical electric field exists, the surface becomes unstable [2]. The calculated critical charge does not exceed the charge falling from the plasma onto the liquid surface, which is roughly estimated as the product of the pulse duration and the conduction current $\sim 10$ A from the oscillogram of the discharge current [1].
The presence of the second, stationary, unstable state can explain why the previous experiments carried [1] only recorded a short-term increase in wettability due to the charge introduced to the meniscus in the form of sharp jumps in the height of the liquid column at the moments of current pulses.

\section{CONCLUSIONS}

A simple mathematical model of the stationary state of the three-phase system "liquid-capillary-gas" was used to demonstrate the role of meniscus electrification in the mechanism of the plasma capillary effect. The results of this work may be useful in the further development of the theory of the plasma capillary effect within the framework of a self-consistent formulation of mechanical and electrostatic problems for a liquid column in a capillary.

\section{REFERENCES}

1. Dubinov, A.E., Kozhayeva, Yu.P., and Selemir, V.D., High Temp., 2018, vol. 56, no. 3, p. 451.

2. Zhakin, A.I., Phys.-Usp., 2013, vol. 56, no. 2, p. 141.

3. Koopal, L.K., Adv. Colloids Interface Sci., 2012, vols. 179182 , p. 29.

4. Mirolyubov, N.N., Kostenko, M.V., Levinshtein, M.L., and Tikhodeev, N.N., Metody rascheta elektrostaticheskikh polei (Methods for Calculating Electrostatic Fields), Moscow: Vysshaya Shkola, 1963.

5. Matubayasi, N., Surface Tension and Related Thermodynamic Quantities of Aqueous Electrolyte Solutions, Boca Ration: CRC, 2013.

https://doi.org/10.1201/b15514 